\title{
Pretreatment neutrophil to lymphocyte and platelet to lymphocyte ratios as predictive factors for the survival of cervical adenocarcinoma patients
}

This article was published in the following Dove Press journal:

Cancer Management and Research

Joanna Jonska-Gmyrek'

Leszek Gmyrek²

Agnieszka Zolciak-Siwinska ${ }^{3}$

Maria Kowalska ${ }^{4}$

Malgorzata Fuksiewicz ${ }^{4}$

Beata Kotowicz ${ }^{4}$

'Department of Uro-oncology, Maria Sklodowska-Curie Institute Oncology Center, Warsaw 02-78I, Poland; ${ }^{2}$ Department of Gynecological Oncology, The Holy Family Hospital, Warsaw 02-544, Poland; ${ }^{3}$ Department of Brachytherapy, Maria SklodowskaCurie Institute - Oncology Center, Warsaw 02-78I, Poland; ${ }^{4}$ Laboratory of Tumor Markers, Department of Pathology and Laboratory Diagnostics, Maria Sklodowska-Curie Institute Oncology Center, Warsaw 02-78I, Poland
Correspondence: Joanna Jonska-Gmyrek Department of Uro-oncology, Maria Sklodowska-Curie Institute - Oncology Center, Roentgen Street 5, Warsaw 02-78I, Poland

Tel +4850 8I 2233I

Email jonska@wp.pl
Objective: Our study assessed the clinical utility and prognostic value of pretreatment hematological parameters and calculated coefficients including the platelet to lymphocyte ratio (PLR), neutrophil to lymphocyte ratio (NLR), and monocyte to lymphocyte ratio (MLR) in patients with cervical adenocarcinoma (CA).

Materials and methods: Among 738 cervical cancer patients with International Federation of Gynecology and Obstetrics (FIGO) stages IA-IV treated at our institution, 96 (13\%) presented with CA histology. The blood samples, collected within 10 days before treatment, were analyzed using a Sysmex XN-2000 system. The statistical tests included Mann-Whitney $U$-tests, log-rank tests, and Cox regression models. The cutoff points for the calculated hematological coefficients (NLR, PLR, and MLR) were determined using the MedCalc statistical program.

Results: The prognostic factor for overall survival (OS) and recurrence-free survival (RFS) in CA was clinical stage according to FIGO classification (FIGO IIB-IV vs I-IIA) $(P=0.0001$; $P=0.002)$. Among patients with FIGO stage IIB-IV treated with radiotherapy/chemoradiotherapy, an elevated PLR was a negative prognostic factor for OS $(P=0.017$; HR: 2.96 ; $95 \%$ CI: 2.069-3.853). Among all patients, an elevated pretreatment NLR was a poor prognostic factor for OS ( $P=0.014$; HR: 2.85 ; 95\% CI: 2.011-3.685) and RFS ( $P=0.049$; HR: 4.0; 95\% CI: 2.612-5.392). The white blood cell count (WBC) before treatment was significantly higher in patients who died during follow-up $(P=0.009)$.

Conclusion: Elevated NLR values before treatment may be associated with a shorter time of RFS and OS, while PLR index may have prognostic significance for OS in patients with advanced disease (FIGO IIB-IV). Both indexes and WBC may be a cost-effective biomarker that can be used conveniently for stratification of recurrence risk and death.

Keywords: platelet to lymphocyte ratio, cervical cancer, neutrophil to lymphocyte ratio, prognostic factors

\section{Introduction}

Due to the development of screening programs, the incidence of cervical cancer has decreased worldwide; however, the incidence of cervical adenocarcinoma (CA) has increased, especially in younger age groups. According to the literature, cervical cancer is the fourth most frequently occurring malignancy among women and the second most common malignancy of the female genital tract, with 200,000 deaths and 500,000 new cases worldwide annually. ${ }^{1,2} \mathrm{CA}$ histopathology accounts for 5\%-25\% of all cervical cancer cases. Data on the influence of histopathology on survival in 
cancer patients are conflicting; however, most studies confirm adenocarcinoma histopathology as a poor prognostic factor in cervical cancer. ${ }^{3-5}$ Some authors have identified different molecular profiles between histopathological types and the need for clinical trials to determine the optimal treatment regimen to enhance the outcome of patients with $\mathrm{CA} .{ }^{4-7}$ Cervical cancer is staged according to International Federation of Gynecology and Obstetrics (FIGO) classification. The clinical stage of the disease can be assessed clinically but other parameters are assessed surgically. Therefore, non-invasive pretreatment parameters, which can be used conveniently and cost-effectively, are needed to predict the clinical course of the disease and the likelihood of response to treatment. Serum C-reactive protein (CRP) is not routinely evaluated before treatment, and it has been shown to have a negative prognostic value in patients with other malignancies such as hepatocellular cancer ${ }^{8}$ and ovarian carcinoma. ${ }^{9}$ The inflammation process plays an important role in the carcinogenesis and cancer progression and may act as a measure of disease progression. Neutrophils have been shown to secrete vascular endothelial growth factor, IL-18, and matrix metalloproteinases that directly contribute to tumor-related angiogenesis, tumor growth, and metastatic processes. ${ }^{10-12}$ Systemic nonspecific markers of inflammation such as the platelet to lymphocyte ratio (PLR) and neutrophil to lymphocyte ratio (NLR) have been investigated in different types of malignancies such as non-small-cell lung cancer, ${ }^{13}$ pancreatic cancer, ${ }^{14}$ gastric cancer, ${ }^{15,16}$ renal cell, ${ }^{17}$ and colorectal cancer. ${ }^{18-20}$ Several studies have also evaluated the role of systemic inflammation in malignancy in cervical cancer. However, little is known about the prognostic value of the pretreatment levels of inflammatory markers, including white blood cell count (WBC), NLR, and PLR, in patients with $\mathrm{CA}$. To our knowledge, there are no studies on the role of these parameters in this histopathological type of cervical carcinoma. Taking into account that adenocarcinoma often presents with less favorable outcomes, ${ }^{3-5,21}$ the assessment of easily applicable prognostic factors for possible additional therapies is of particular importance.

The aim of this study was to determine the clinical usefulness and prognostic value of pretreatment hematological parameters including WBC, hemoglobin $(\mathrm{Hb})$, and platelets (PLT) as well as calculated coefficients such as NLR, PLR, and monocyte to lymphocyte ratio (MLR) in patients with CA.

\section{Materials and methods}

We retrospectively analyzed data from cervical cancer patients with FIGO stage IA-IV disease who were treated consecutively from November 2003 to January 2008 at the Maria Sklodowska-Curie Institute-Oncology Center in Warsaw. The eligibility criteria included patients with cervical cancer with confirmed adenocarcinoma histopathology who were qualified to receive radical treatment. The histopathological features of all tumors were confirmed by our institutional pathologist. Data on all clinical factors and complete blood parameters were collected within 10 days before treatment. Blood samples were evaluated using a Sysmex XN-2000 system. The NLR was defined as the absolute number of neutrophils divided by the absolute number of lymphocytes (G/L), the PLR as the absolute number of PLT by the absolute number of lymphocytes $(\mathrm{G} / \mathrm{L})$, and the MLR as the absolute number of monocytes by the absolute number of lymphocytes $(\mathrm{G} / \mathrm{L})$ in patients with adenocarcinoma of the uterine cervix. Patients were staged according to FIGO classification and treated according to European Society for Medical Oncology and European Society of Radiotherapy and Oncology (ESTRO) recommendations. Before treatment, patients were staged with clinical examination, computed tomography (CT) or X-ray imaging of the chest, and CT or magnetic resonance (MR) of the abdomen and pelvis. In case of suspected infiltration of the bladder or rectum, histopathological confirmation of cystoscopy or rectoscopy specimens was performed. Patients with early cervical cancer (FIGO stages IA 1-2, IB1, and IIA) were treated with radical hysterectomy and bilateral oophorectomy, including the part of the cardinal and uterosacral ligament at the upper end of the vagina, as well as pelvic lymphadenectomy. In case of enlarged para-aortal lymph nodes, para-aortal lymphadenectomy or biopsy was performed. After surgical treatment, patients were qualified for adjuvant therapy based on risk factors. In case of the presence of at least two intermediate risk factors such as deep stromal invasion, large tumor, or lymphovascular space invasion, the patients were administered external beam irradiation (EBRT) with or without chemotherapy and brachytherapy (BT) as two 7.5-Gray (Gy) fractions prescribed to the vaginal vault. Patients with high-risk factors such as positive lymph nodes (N1), positive margins (R1), or parametrial infiltration were treated with chemoradiotherapy (CCRT). Patients with locally advanced cervical cancer, FIGO stages IB2, IIB-IVA, were also treated with CCRT. The first-line therapy in these patients included CCRT with weekly cisplatin at a dose of $40 \mathrm{mg} / \mathrm{m}^{2}$ of body surface area. Patients qualified to receive CCRT were treated with EBRT to the pelvis, and sometimes, to the para-aortic area, based on the three-dimensional (3D) planning system calculated using a HELAX system, according to the recommendations 
of Reports 50 and 62 of the International Commission on Radiation Units and Measurements (ICRU) with a 3D conformal technique. Clinical target volumes (CTVs) with a margin of $5 \mathrm{~mm}$ in all directions were delineated on the CT scans, and the planning target volumes (PTVs) were defined. Organs at risk (OAR) were also delineated during the planning procedure. Patients were treated with a total dose of 45-46 Gy to the pelvic and/or para-aortal region, and a 60-64 Gy boost in cases of enlarged lymph nodes measuring over $2 \mathrm{~cm}$ or resistant tumor in the parametrium. The patients started high-dose rate (HDR) BT in 1-3 weeks after EBRT. BT was performed with an iridium (Ir-192) source from microSelectron $^{\circledR}$ (Nucletron Devices) once a week with 4 fractions of $7.5 \mathrm{~Gy}$ for a total dose of $30 \mathrm{~Gy}$ prescribed to the high-risk CTV. BT was planned according to the Groupe Européen de Curiethérapie-ESTRO recommendations with wider margins in the uterus and vaginal directions due to the lack of MR-based planning possibilities, according to the ICRU 38 Report. The planning system was based on CT images or radiographs. During the planning procedure, the PTV and OAR were delineated on CT scans. Fletcher, ring applicator, or 2-6 interstitial metal needles and intrauterine catheters were used to perform the HDR-BT.

The patients were followed-up every 3 months for the first 2 years, at 6-month intervals for the next 3, and annually after 5 years. In case of suspected relapse, we obtained histologically evaluated biopsy specimens. Patient follow-up was maintained until death or last observation, with a cutoff date of December 31, 2017.

Nonparametric Mann-Whitney $U$-tests were used for statistical comparisons of two independent groups. The probabilities of recurrence-free survival (RFS) and overall survival (OS) were assessed using univariate analysis with log-rank tests and multivariate analysis using Cox proportional hazards models. The OS was defined as the time interval between the start of treatment and the last follow-up or death. The RFS was the period between the first day of the treatment and disease recurrence, local or distant. The level of significance was $P<0.05$. The cutoff points for the calculated hematological coefficients (NLR, PLR, and MLR) were determined using the MedCalc statistical program.

\section{Results}

Among 738 patients with cervical cancer treated at our center, we excluded those with squamous cell histopathology (642) and two CA patients treated with palliative intent and included all patients with $\mathrm{CA}$ who were qualified to receive radical treatment; thus, a total of 94 patients with CA were included in the final analysis. Their median age was 53 years (range: 20-81 years). Forty-two patients had early-stage disease (FIGO I-IIA) and 52 had advanced disease (FIGO IIB-IVA). Early-stage disease was confirmed in $37(61 \%)$ premenopausal and 57 (35\%) postmenopausal patients. During the long-term follow-up (median: 66 months; range: 4.6-171.4 months), disease recurrence was observed in 44 (49\%) patients (median age: 54 years), including 15 premenopausal and 29 postmenopausal women. The median time to relapse was 25 months. Forty-eight (51\%) patients died. The clinical and pathological features of the patients with CA, the treatment characteristics, and the outcomes are presented in Table 1.

\section{NLR, PLR, and MLR and the ranges of the absolute numbers and median hematological parameters}

The median pretreatment NLR was 2.32 (range: 0.7-20.3). The median PLR was 158 (range: 62.6-634). The calculated coefficients of NLR, PLR, and MLR, the ranges of the absolute numbers, and medians of the hematological parameters ( $\mathrm{Hb}, \mathrm{WBC}$, and PLT) overall according to FIGO and clinical conditions are presented in Table 2.

We next evaluated the correlation between the examined parameters and the age of patients, FIGO stage, and clinical status during the follow-up. No statistically significant correlations between age ( $<50 \mathrm{vs}>50$ years), FIGO stage, and the values of the studied parameters were observed. In patients with FIGO IIB-IV disease, only a trend of elevated NLR was observed $(P=0.06)$. Of all hematological parameters, pretreatment WBC $(P=0.009)$ was significantly higher in patients who died during follow-up than in patients who survived, as analyzed using the Mann-Whitney $U$-test (Figure 1). Higher WBC values were also demonstrated in patients with metastases compared to the nonmetastatic group, however, this relationship was observed to be a trend $(P=0.054)$.

Based on the analysis of receiver operating characteristic (ROC) curves for hematological factors cutoff points for NLR, PLR, and MLR were determined while maintaining the optimal sensitivity and specificity of tests for recurrence and no-recurrence groups. The results are presented in Table 3.

Next, we analyzed the prognostic value of the examined parameters for RFS and OS. The first group comprised of patients treated with surgery and BT, EBRT, or CCRT $(n=47)$; and the second group included patients treated with radiotherapy (RT) alone or CCRT $(n=47)$. 
Table I Patients, treatment, and clinical outcome characteristics

\begin{tabular}{|c|c|c|}
\hline Parameter & Feature & $\begin{array}{l}\text { Number of } \\
\text { patients (\%) } \\
94(100 \%)\end{array}$ \\
\hline Age, years & Median (range) & $53(20-8 I)$ \\
\hline MS & $\begin{array}{l}\text { Premenopausal } \\
\text { Postmenopausal }\end{array}$ & $\begin{array}{l}37(39) \\
57(61)\end{array}$ \\
\hline FIGO stage & $\begin{array}{l}\text { IA } \\
\text { IB } \\
\text { IIA } \\
\text { IIB } \\
\text { IIIA } \\
\text { IIIB } \\
\text { IVA } \\
\text { IVB }\end{array}$ & $\begin{array}{l}2(2) \\
33(35) \\
7(7.5) \\
24(25.5) \\
0(0) \\
26(28) \\
I(1) \\
I(I)\end{array}$ \\
\hline AJCC stage & $\begin{array}{l}\text { TIBNOM0 } \\
\text { TIBNIM0 } \\
\text { T2NOM0 } \\
\text { T2NIM0 } \\
\text { T2NOMI } \\
\text { T3NOM0 } \\
\text { T3NIM0 } \\
\text { T3NIMI } \\
\text { T4NOM0 } \\
\text { T4NIM0 }\end{array}$ & $\begin{array}{l}33(35) \\
2(2) \\
24(26) \\
7(7.5) \\
0(0) \\
18(19) \\
7(7.5) \\
1(1) \\
I(1) \\
I(1)\end{array}$ \\
\hline Histology & $\begin{array}{l}\text { Pure adenocarcinoma } \\
\text { Papillare } \\
\text { Adenosquamous } \\
\text { Mucinous } \\
\text { Endometrioides } \\
\text { Clear cell } \\
\text { Small cell } \\
\text { Villoglandular } \\
\end{array}$ & $\begin{array}{l}54(57.5) \\
10(11) \\
6(6) \\
11(12) \\
4(4) \\
7(7.5) \\
1(1) \\
1(1) \\
\end{array}$ \\
\hline Tumor grade & $\begin{array}{l}\mathrm{Gl} \\
\mathrm{G} 2 \\
\mathrm{G} 3 \\
\mathrm{Gx}\end{array}$ & $\begin{array}{l}23(24.5) \\
39(41.5) \\
12(13) \\
20(2 I)\end{array}$ \\
\hline $\mathrm{LN}$ & $\begin{array}{l}\mathrm{N}+ \\
\mathrm{N}- \\
\mathrm{ND}\end{array}$ & $\begin{array}{l}18(19) \\
68(72) \\
8(9) \\
\end{array}$ \\
\hline Treatment & $\begin{array}{l}\text { Surgery } \\
\text { Surgery + BT } \\
\text { Surgery + CCRT + BT } \\
\text { Surgery + EBRT + BT } \\
\text { CCRT } \\
\text { EBRT }\end{array}$ & $\begin{array}{l}7(7.5) \\
5(5.3) \\
22(23.4) \\
13(13.8) \\
45(48) \\
2(2)\end{array}$ \\
\hline EBRT & $\begin{array}{l}46 \mathrm{~Gy} / 23 \mathrm{fr} \\
45 \mathrm{~Gy} / 25 \mathrm{fr} \\
45.9 \mathrm{~Gy} / 27 \mathrm{fr} \\
50.4 \mathrm{~Gy} / 28 \mathrm{fr} \\
50 \mathrm{~Gy} / 25 \mathrm{fr} \\
\text { Boost delivered to enlarged } \\
\text { lymph nodes over } 2 \mathrm{~cm} \text { in } \\
\text { short axis } 59.4 \mathrm{~Gy} / 27 \mathrm{fr} \text { to } \\
60 \mathrm{~Gy} / 27 \mathrm{fr}(\mathrm{SIB}) \\
64 \mathrm{~Gy} / 23 \mathrm{fr}+\text { boost } \\
\text { delivered to U\&P }\end{array}$ & $\begin{array}{l}49(52) \\
7(7) \\
1(1) \\
0 \\
7(7) \\
6(6)\end{array}$ \\
\hline
\end{tabular}

(Continued)
Table I (Continued)

\begin{tabular}{|l|l|l|}
\hline Parameter & Feature & $\begin{array}{l}\text { Number of } \\
\text { patients (\%) } \\
\mathbf{9 4}(\mathbf{1 0 0 \% )}\end{array}$ \\
\hline BT HDR & $30 \mathrm{~Gy} / 4 \mathrm{fr}$ & $25(24)$ \\
BT LDR & $22.5 \mathrm{~Gy} / 3 \mathrm{fr}$ & $7(7)$ \\
& I5 Gy/2 fr & $25(27)$ \\
& $28 \mathrm{~Gy} / 4 \mathrm{fr}$ & $2(2)$ \\
& $45 \mathrm{~Gy} / 2 \mathrm{fr}$ & $7(7)$ \\
\hline BT & LDR & $7(7)$ \\
& HDR & $59(63)$ \\
& RSA & $40(43)$ \\
& IT & $17(40)$ \\
& FA & $22(50)$ \\
& T\&R & $4(10)$ \\
\hline CHTH (DDP) & I-3 courses & $5(1 \mathrm{I})$ \\
& $4-6$ courses & $40(89)$ \\
\hline Recurrence & Yes & $44(47)$ \\
& No & $50(53)$ \\
\hline Age of & Median (range) & $54(26-85)$ \\
recurrence & & \\
\hline Recurrence & Premenopausal & $15(34)$ \\
AMS & Postmenopausal & $29(65)$ \\
\hline AF & I-IIA & $13(30)$ \\
& IIB-IV & $3 I(70)$ \\
\hline Death & Yes & $48(5 \mathrm{I})$ \\
& No & $46(49)$ \\
\hline Age of death & Median (range) & $54(26-85)$ \\
\hline Death AMS & Premenopausal & $31(35)$ \\
& Postmenopausal & $12(25)$ \\
\hline Death AF & I-IIA & $36(75)$ \\
\hline IIB-IV & \\
\hline
\end{tabular}

Abbreviations: AF, according to FIGO; AJCC, American Joint Committee on Cancer; AMS, according to menopausal status; BT, brachytherapy; CCRT, radiochemotherapy; $\mathrm{CHTH}$, chemotherapy; DDP, cisplatin; EBRT, external beam radiation therapy; FA, Fletcher applicator; FIGO, International Federation of Gynecology and Obstetrics; fr, fraction; HDR, high-dose rate; IT, interstitial; LDR, low-dose rate; LN, lymph nodes; MS, menopausal status; ND, no data; RSA, rollershaped applicator; SIB, simultaneous integrated boost; T\&R, tandem and ring; U\&P, uterus and parametria.

\section{The influence of the examined}

\section{parameters on RFS in the whole group of patients}

The results of the log-rank univariate analysis demonstrated that disease stage (FIGO IIB-IV) $(P=0.002)$ and conservative treatment modality (patients treated only with RT or CCRT) $(P=0.004)$ were associated with a shorter time to relapse. Among the hematological coefficients, NLR tended to be associated with RFS $(P=0.059)$. However, multivariate Cox analysis demonstrated that the rate of NLR $(P=0.021$; HR 3.0; 95\% CI: 2.063-3.936) was an independent prognostic factor and elevated values were associated with a worse RFS (Figure 2A). In the group of patients treated with surgery and 
Table 2 Median values and concentration ranges for NLR, PLR, MLR, WBC, Hb, and PLT according to the clinical condition

\begin{tabular}{|c|c|c|c|c|c|c|}
\hline Patient groups & NLR & PLR & MLR & WBC, G/L & Hb, G/dL & PLT, G/L \\
\hline All patients & $\begin{array}{l}2.32 \\
(0.7-20.3)\end{array}$ & $\begin{array}{l}158 \\
(62.6-634)\end{array}$ & $\begin{array}{l}0.31 \\
(0.13-1 \mid .2)\end{array}$ & $\begin{array}{l}6.6 \\
(1.8-18.6)\end{array}$ & $\begin{array}{l}12.8 \\
(97.7-15.2)\end{array}$ & $\begin{array}{l}279 \\
(|43-83|)\end{array}$ \\
\hline FIGO I-IIA & $\begin{array}{l}1.93 \\
(0.7-20.3)\end{array}$ & $\begin{array}{l}149 \\
(80.8-634)\end{array}$ & $\begin{array}{l}0.28 \\
(0.13-\mid 1.2)\end{array}$ & $\begin{array}{l}6.1 \\
(3.9-17.7)\end{array}$ & $\begin{array}{l}12.5 \\
(9.2-14.8)\end{array}$ & $\begin{array}{l}258 \\
(143-509)\end{array}$ \\
\hline FIGO IIB-IV & $\begin{array}{l}2.7 \\
(1.03-9.6)\end{array}$ & $\begin{array}{l}160 \\
(62.6-539)\end{array}$ & $\begin{array}{l}0.33 \\
(0.14-10.2)\end{array}$ & $\begin{array}{l}6.9 \\
(1.8-18.6)\end{array}$ & $\begin{array}{l}12.9 \\
(7.7-15.2)\end{array}$ & $\begin{array}{l}284 \\
(I 56-83 \mid)\end{array}$ \\
\hline Without recurrence & $\begin{array}{l}2.2 \\
(0.8-20.3)\end{array}$ & $\begin{array}{l}156 \\
(80.8-584)\end{array}$ & $\begin{array}{l}0.31 \\
(0.13-11.1)\end{array}$ & $\begin{array}{l}6.1 \\
(1.8-17.7)\end{array}$ & $\begin{array}{l}12.9 \\
(7.8-15.2)\end{array}$ & $\begin{array}{l}256 \\
(143-603)\end{array}$ \\
\hline With recurrence & $\begin{array}{l}2.35 \\
(0.7-\mid I .9)\end{array}$ & $\begin{array}{l}165 \\
(62.6-634)\end{array}$ & $\begin{array}{l}0.32 \\
(0.14-2.1)\end{array}$ & $\begin{array}{l}7.1 \\
(3.9-18.6)\end{array}$ & $\begin{array}{l}12.6 \\
(7.7-15.2)\end{array}$ & $\begin{array}{l}287 \\
(|75-83|)\end{array}$ \\
\hline Died & $\begin{array}{l}2.6 \\
(0.68-11.9)\end{array}$ & $\begin{array}{l}160 \\
(62.6-634)\end{array}$ & $\begin{array}{l}0.31 \\
(0.14-0.91)\end{array}$ & $\begin{array}{l}7.2 \\
(3.9-18.6)\end{array}$ & $\begin{array}{l}12.7 \\
(7.7-15.2)\end{array}$ & $\begin{array}{l}286 \\
(|75-83|)\end{array}$ \\
\hline
\end{tabular}

Abbreviations: FIGO, International Federation of Gynecology and Obstetrics; Hb, hemoglobin; MLR, monocyte to lymphocyte ratio; NLR, neutrophil to lymphocyte ratio; PLR, platelet to lymphocyte ratio; PLT, platelets; WBC, white blood cell count.

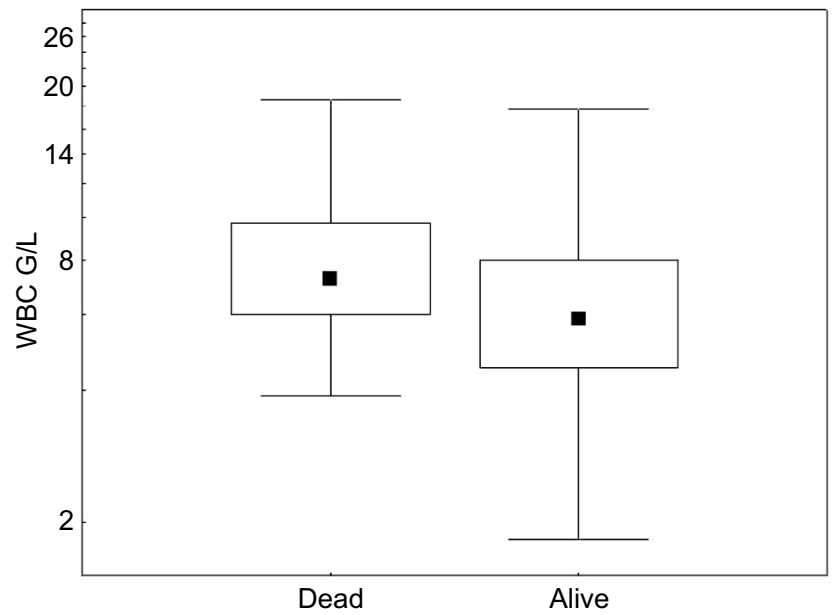

Figure I Medians and WBC values according to clinical status (alive vs dead). Abbreviation: WBC, white blood cell count.

further BT, RT, or CCRT ( $\mathrm{n}=47)$, the correlation between $\operatorname{NLR}(P=0.001)$, PLR $(P=0.006)$, and $\mathrm{Hb}(P=0.01)$ with RFS was demonstrated in log-rank tests, while Cox analysis revealed that only elevated NLR $(P=0.049$; HR: 4.0; 95\% CI: 2.612-5.392) was an independent and poor prognostic factor for RFS (Figure 2B).

\section{The prognostic value of the examined parameters on OS in the whole group of patients}

OS was associated with disease stage (FIGO IIB-IV) $(P=0.0001)$ and conservative treatment modality, which is the treatment of choice for this clinical stage. Patients treated with RT or CCRT only $(P=0.004)$ had a shorter OS, which may also be an effect of the disease advancement. Cox multivariate analysis revealed that, among hematological
Table 3 ROC analysis: NLR, PLR, and MLR test characteristics for remission or recurrence in patients with adenocarcinoma of the cervix

\begin{tabular}{|l|l|l|l|l|}
\hline Parameters & Cutoff & Sensitivity & Specificity & AUC \\
\hline NLR & 1.6 & 88.1 & 31.8 & 0.562 \\
\hline PLR & 158 & 57.1 & 59.1 & 0.527 \\
\hline MLR & 0.33 & 45.2 & 68.2 & 0.511 \\
\hline
\end{tabular}

Abbreviations: AUC, area under the curve; MLR, monocyte to lymphocyte ratio; NLR, neutrophil to lymphocyte ratio; PLR, platelet to lymphocyte ratio; ROC, receiver operating characteristic.

parameters, an elevated NLR was a poor prognostic factor for $\operatorname{OS}(P=0.014 ; \mathrm{HR}: 2.85 ; 95 \% \mathrm{CI}: 2.011-3.685)$ (Figure 3).

Survival analysis in patient groups divided according to FIGO stage and treatment modality confirmed that elevated values of PLR were correlated with poor prognosis for OS in patients with FIGO stage IIB-IV treated with RT/CCRT ( $P=0.017$; HR: 2.96; 95\% CI: 2.069-3.853) (Figure 4).

\section{Discussion}

To our knowledge, this is the first study with mature data to investigate the prognostic value of pretreatment NLR, PLR, and other hematological parameters in patients with CA. As this histopathological type generally has a less favorable outcome, ${ }^{21,22}$ easy and inexpensive identification of high-risk groups is especially important. Therefore, selecting patients with a particularly unfavorable prognosis and encouraging them to consider the use of new forms of treatment is a priority task. This study is a continuation of a material analysis of tumor markers. ${ }^{23}$ Further analyses were performed to identify low-cost and widely available data on hematological parameters in this group of patients. 

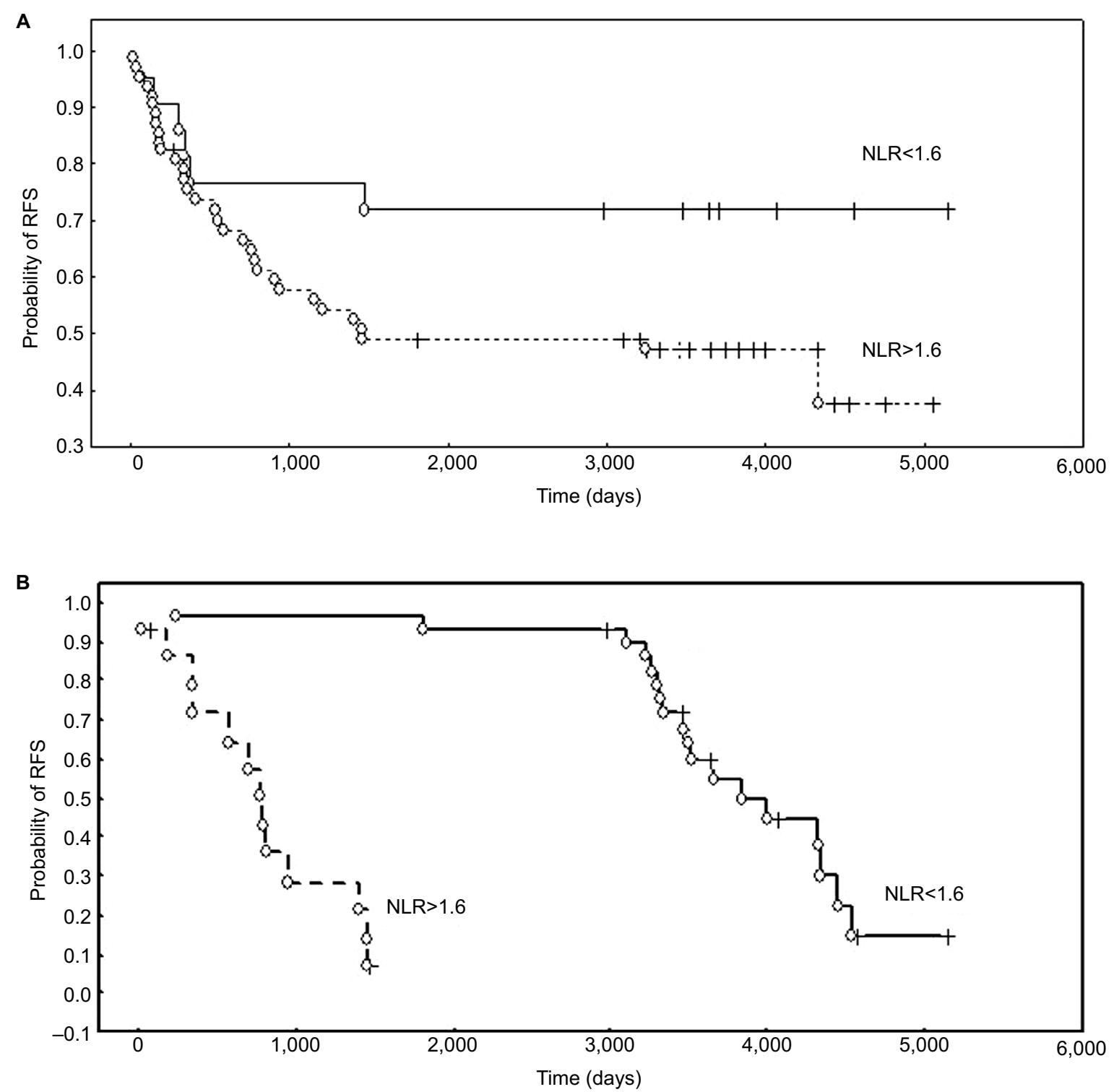

Figure 2 The probability of RFS according to NLR.

Notes: (A) RFS of CA patients treated with RT/CCRT stratified by NLR. (B) Probability of RFS according to NLR in patients treated with primary surgery and adjuvant RT/CCRT.

Abbreviations: CA, cervical adenocarcinoma; CCRT, chemoradiotherapy; NLR, neutrophil to lymphocyte ratio; RFS, recurrence-free survival; RT, radiotherapy.

The NLR may act as a marker for the evaluation of the systemic balance between pro-tumor inflammation associated with neutrophils and antitumor immune response associated with lymphocytes. An increased NLR may indicate a trend for decreased antitumor immune capacity and increased pro-tumor inflammation. ${ }^{24}$ In another study, ${ }^{25}$ a higher NLR was associated with more advanced disease stage in younger patients. More advanced disease stage and elevated pretreatment NLR in patients with CA in our study were significantly associated with poorer RFS and OS. This finding is in accordance with previous studies reporting that the pretreatment NLR is associated with survival in patients with other malignancies as well as cervical cancer, especially those with advanced disease. ${ }^{26,27}$ Zhang et al demonstrated the poor prognostic influence of NLR on RFS, but not OS, in cervical carcinoma patients treated with surgery. They found that an elevated NLR was associated with lymph node metastases (LNM) and the depth of stromal infiltration. ${ }^{28}$ In patients with cervical squamous cell carcinoma treated with CCRT, NLR was a highly significant prognostic factor for RFS and OS. Moreover, a low NLR was significantly associated with a complete response to treatment with CCRT. ${ }^{29}$ In an analysis by Huang et al, a high pretreatment NLR was an adverse prognostic factor for OS and RFS and was also significantly 


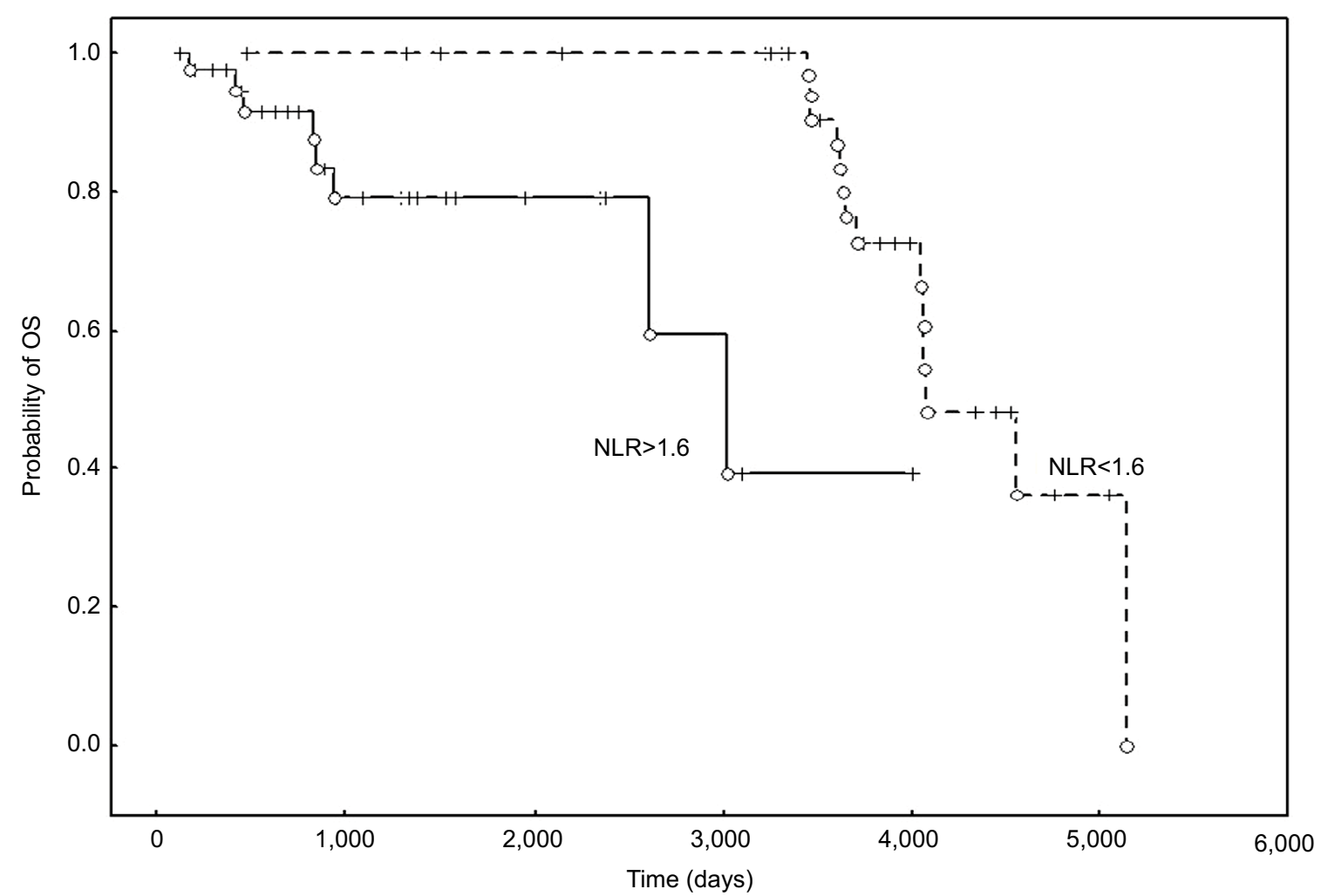

Figure 3 OS of CA patients stratified by NLR.

Abbreviations: CA, cervical adenocarcinoma; NLR, neutrophil to lymphocyte ratio; OS, overall survival.

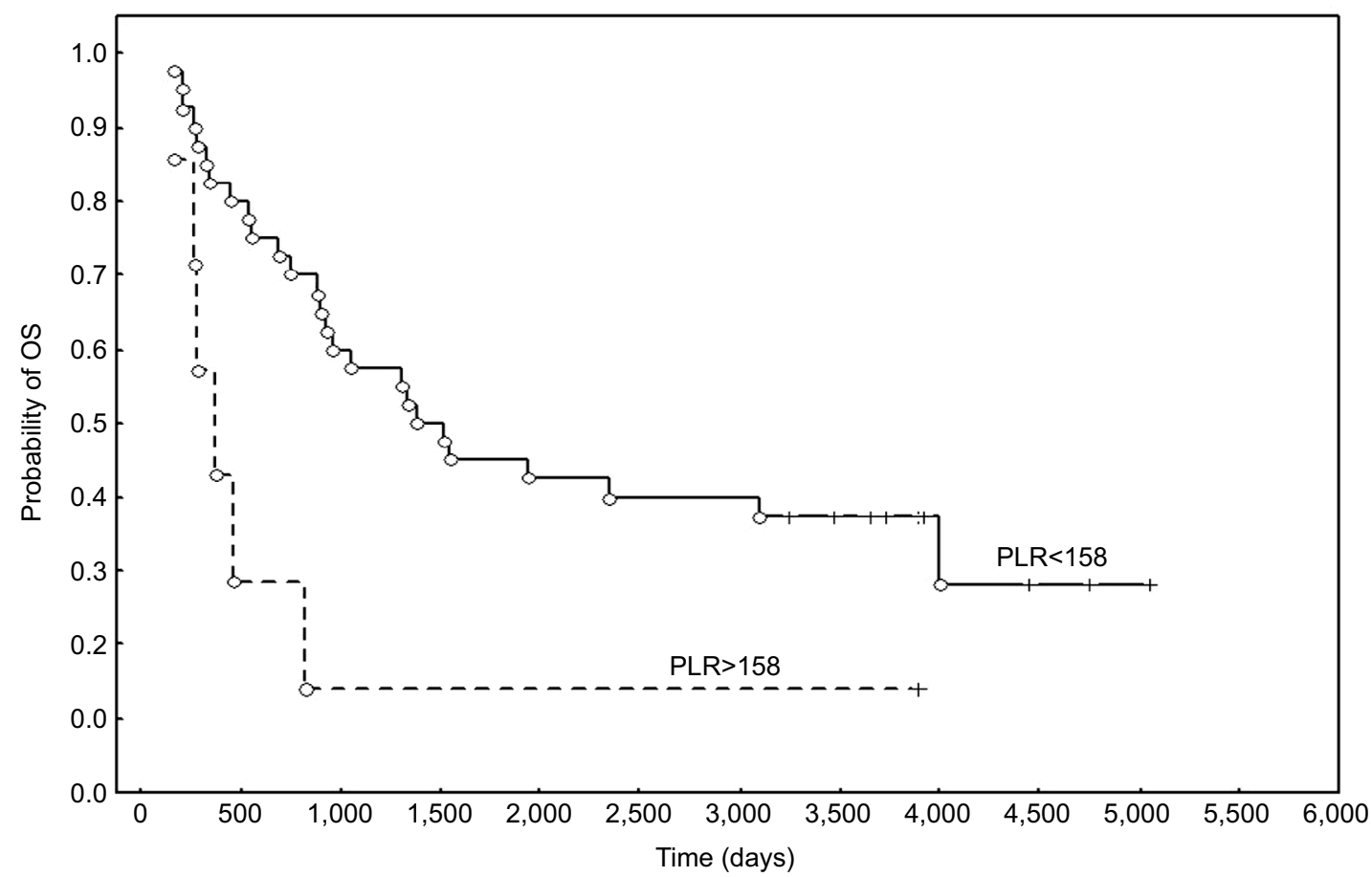

Figure 4 The probability of OS according to the PLR in patients with FIGO stage IIB-IV treated with RT/CCRT.

Abbreviations: CCRT, chemoradiotherapy; FIGO, International Federation of Gynecology and Obstetrics; OS, overall survival; PLR, platelet to lymphocyte ratio; RT, radiotherapy. 
associated with FIGO stage, LNM, and tumor size. ${ }^{30}$ The prognostic value of pretreatment NLR was confirmed in a study of patients with a wide range of malignancies. ${ }^{31}$ In a study of cervical cancer treated with RT, Cho et al reported that an increased NLR was associated with a poorer treatment response and more aggressive disease. ${ }^{32}$

Our results also confirmed that more advanced FIGO stage and elevated NLR were significantly associated with a poorer outcome. Wang et al did not confirm the predictive values of NLR and PLR in cervical cancer patients treated with neoadjuvant chemotherapy and surgery. ${ }^{33}$ However, our study did not include these patients.

Besides low lymphocyte values, thrombocytosis has also been reported as a marker of host systemic inflammation. Its prognostic value in malignancies has been confirmed. ${ }^{34-37}$ Pretreatment peripheral PLR has been shown to have prognostic value for LNM and clinical outcome in early cervical cancer treated with surgery. ${ }^{38}$ According to Nakamura et al, the pretreatment PLR may be an independent predictor of recurrence in cervical cancer patients treated with CCRT. ${ }^{39}$ The findings of our study demonstrated that pretreatment PLR was significantly correlated with the prognosis of CA patients treated with RT/CCRT. Elevated pretreatment PLR in patients with FIGO stage IIB-IV disease treated with RT/CCRT negatively impacted the OS. Once confirmed in a larger study group, these results have significant potential for wider application in practice. Considering that CA histology has been reported to be a poor prognostic factor for worse response to CCRT, this is a unique information with regard to this group of patients. Therefore, it seems reasonable to consider whether this particular group should not be subjected to complementary treatments such as targeted therapy or the addition of anti-inflammatory agents.

Leukocytes, being at the center of the inflammatory response to tumor formation, have been the subject of evaluation in many studies on colorectal and lung cancers. ${ }^{12,40}$ The results of our study demonstrated that elevated pretreatment values of WBC in patients with cervical carcinoma were related to a shorter OS. The findings of other study confirmed that WBC may predict survival in these patients. The prognostic value of WBC in cervical cancer has been demonstrated in other studies. ${ }^{25,34,41}$ The total WBC may be a significant addition to the other established markers of systemic inflammation, including albumin, CRP, lymphocytes counts, neutrophil, prognostic nutritional index, and their combination. ${ }^{26,42,43}$ The results of our study suggest that inflammatory components such as lymphocytes, PLT, neutrophils, and WBC play a critical role in the development and progression of CA. The specific relationships between these components and poor survival are yet to be elucidated. However, pretreatment lymphocyte and neutrophil numbers indicate the level of systemic inflammation. ${ }^{44-46}$ Inflammation may change the tumor microenvironment and lead to promotion of angiogenesis and metastasis..$^{25,47,48}$ Moreover, circulating lymphocytes may secrete cytokines that play a role in preventing proliferation and metastatic process and in cytotoxicity. Lower lymphocyte levels and NLR imbalance may promote progression of malignancy. More advanced cancer stage may significantly affect hematological parameters, unlike smaller tumors. Thus, the NLR reflects the balance between inflammatory and immune responses. ${ }^{19,49}$ Moreover, more advanced FIGO stage (FIGO IIB-IVA) in patients with $\mathrm{CA}$ is a poor prognostic factor for OS; in our study, treatment with RT/CCRT was related to a poor prognosis, probably related to the disease severity. However, we have to consider that this is the treatment of choice for this group of patients, and our study aimed to identify inexpensive markers that can be conveniently used for patient identification. Based on the results of the studies available in the literature, and analyzing the relationship between the value of the assessed parameters and the survival of patients, we can conclude that they may have a prognostic value. We also know that the inflammation process may promote the cancer progression. We assume that an unfavorable prognosis of patients with adenocarcinoma of the cervix is not a result of the increased value of the analyzed parameters. Elevated parameters seem to be a symptom of cancer advancement and evidence that a value of coefficients reflects the fact that after exceeding a limit, the tumor mass is so high that the chances for a favorable treatment outcome are drastically reduced. Future studies are necessary to assess additive, alternative, or more effective treatment regimens.

The strength of our study was the large group of patients with CA and the long follow-up, which presented mature results. To our knowledge, this is the first report on the influence of inflammatory parameters on the outcome of patients with CA. The weakness of our study is its retrospective nature and single institution experience. However, if proven in future clinical studies, WBC, NLR, and PLR might be novel, relatively easily available, and inexpensive prognostic markers that help in guiding therapeutic methods and identifying CA patients with high-risk profiles. A histological type, characterized by a poorer prognosis among patients with cervical cancer and concerning an increasingly younger population, our study identified high-risk patients with CA FIGO IIBIV and elevated WBC, NLR, and PLR before treatment. If confirmed in larger cohorts, these hematological parameters 
and ratios may identify patients who may respond to antiinflammatory treatment.

\section{Conclusion}

To our knowledge, this is the largest report of CA patients with mature data. We first demonstrated the prognostic significance of pretreatment PLR, NLR, and WBC values on the survival of patients with CA. Furthermore, the combination of NLR and PLR may enable risk stratification for survival prediction. These parameters can be derived directly from routine blood cell counts, making it an easily available marker for individual risk assessment and stratification of patients with uterine $\mathrm{CA}$, in addition to the clinical stage. Changes in blood NLR and PLR might have utility in tailored therapy, especially in advanced stages that lack reliable markers.

\section{Author contributions}

All authors contributed to data analysis, drafting or revising the article, gave final approval of the version to be published, and agree to be accountable for all aspects of the work.

\section{Disclosure}

The authors report no conflicts of interest in this work.

\section{References}

1. Bodner-Adler B, Kimberger O, Schneidinger C, Kölbl H, Bodner K. Prognostic significance of pre-treatment serum C-reactive protein level in patients with adenocarcinoma of the uterine cervix. Anticancer Res. 2016;36(9):4691-4696.

2. Small W, Bacon MA, Bajaj A, et al. Cervical cancer: a global health crisis. Cancer. 2017;123(13):2404-2412.

3. Yokoi E, Mabuchi S, Takahashi R, et al. Impact of histological subtype on survival in patients with locally advanced cervical cancer that were treated with definitive radiotherapy: adenocarcinoma/adenosquamous carcinoma versus squamous cell carcinoma. J Gynecol Oncol. 2017;28(2):e19.

4. Rose PG. Are the differences in treatment outcome for adenocarcinoma of the cervix different enough to change the treatment paradigm? Gynecol Oncol. 2012;125(2):285-286.

5. Williams NL, Werner TL, Jarboe EA, Gaffney DK. Adenocarcinoma of the cervix: should we treat it differently? Curr Oncol Rep. 2015;17(4):17.

6. Wright AA, Howitt BE, Myers AP, et al. Oncogenic mutations in cervical cancer: genomic differences between adenocarcinomas and squamous cell carcinomas of the cervix. Cancer. 2013;119(21): 3776-3383.

7. Tornesello ML, Buonaguro L, Buonaguro FM. Mutations of the TP53 gene in adenocarcinoma and squamous cell carcinoma of the cervix: a systematic review. Gynecol Oncol. 2013;128(3):442-448.

8. Gomez D, Farid S, Malik HZ, et al. Preoperative neutrophil-tolymphocyte ratio as a prognostic predictor after curative resection for hepatocellular carcinoma. World J Surg. 2008;32(8):1757-1762.

9. Hefler LA, Concin N, Hofstetter G, et al. Serum C-reactive protein as independent prognostic variable in patients with ovarian cancer. Clin Cancer Res. 2008;14(3):710-714.
10. Jablonska E, Puzewska W, Grabowska Z, Jablonski J, Talarek L. VEGF, IL-18 and NO production by neutrophils and their serum levels in patients with oral cavity cancer. Cytokine. 2005;30(3):93-99.

11. Ardi VC, Kupriyanova TA, Deryugina EI, Quigley JP. Human neutrophils uniquely release TIMP-free MMP-9 to provide a potent catalytic stimulator of angiogenesis. Proc Natl Acad Sci U S A. 2007;104(51):20262-20267.

12. Di Carlo E, Forni G, Musiani P. Neutrophils in the antitumoral immune response. Chem Immunol Allergy. 2003;83:182-203.

13. Yin Y, Wang J, Wang X, et al. Prognostic value of the neutrophil to lymphocyte ratio in lung cancer: A meta-analysis. Clinics. 2015;70(7):524-530.

14. Sugiura T, Uesaka K, Kanemoto H, Mizuno T, Okamura Y. Elevated preoperative neutrophil-to-lymphocyte ratio as a predictor of survival after gastroenterostomy in patients with advanced pancreatic adenocarcinoma. Ann Surg Oncol. 2013;20(13):4330-4337.

15. Lee S, Oh SY, Kim SH, et al. Prognostic significance of neutrophil lymphocyte ratio and platelet lymphocyte ratio in advanced gastric cancer patients treated with FOLFOX chemotherapy. BMC Cancer. 2013;13:350.

16. Jin $\mathrm{H}$, Zhang G, Liu X, et al. Blood neutrophil-lymphocyte ratio predicts survival for stages III-IV gastric cancer treated with neoadjuvant chemotherapy. World J Surg Oncol. 2013;11:112.

17. Pichler M, Hutterer GC, Stoeckigt C, et al. Validation of the pretreatment neutrophil-lymphocyte ratio as a prognostic factor in a large European cohort of renal cell carcinoma patients. Br J Cancer. 2013;108(4):901-907.

18. Jankova L, Dent OF, Chan C, Chapuis P, Clarke SJ. Preoperative neutrophil/lymphocyte ratio predicts overall survival but does not predict recurrence or cancer-specific survival after curative resection of nodepositive colorectal cancer. BMC Cancer. 2013;13:442.

19. Ding PR, An X, Zhang RX, et al. Elevated preoperative neutrophil to lymphocyte ratio predicts risk of recurrence following curative resection for stage IIA colon cancer. Int J Colorectal Dis. 2010;25(12):1427-1433.

20. Galon J, Costes A, Sanchez-Cabo F, et al. Type, density, and location of immune cells within human colorectal tumors predict clinical outcome. Science. 2006;313(5795):1960-1964.

21. Jung EJ, Byun JM, Kim YN, et al. Cervical adenocarcinoma has a poorer prognosis and a higher propensity for distant recurrence than squamous cell carcinoma. Int J Gynecol Cancer. 2017;27(6):1228-1236.

22. Galic V, Herzog TJ, Lewin SN, et al. Prognostic significance of adenocarcinoma histology in women with cervical cancer. Gynecol Oncol. 2012;125(2):287-291.

23. Kotowicz B, Kaminska J, Fuksiewicz M, et al. Clinical significance of serum CA-125 and soluble tumor necrosis factor receptor type I in cervical adenocarcinoma patients. Int $J$ Gynecol Cancer. 2010;20(4):588-592.

24. Moses K, Brandau S. Human neutrophils: Their role in cancer and relation to myeloid-derived suppressor cells. Semin Immunol. 2016;28(2):187-196.

25. Lee YY, Choi CH, Kim HJ, et al. Pretreatment neutrophil:lymphocyte ratio as a prognostic factor in cervical carcinoma. Anticancer Res. 2012;32(4):1555-1561.

26. Proctor MJ, McMillan DC, Morrison DS, Fletcher CD, Horgan PG, Clarke SJ. A derived neutrophil to lymphocyte ratio predicts survival in patients with cancer. Br J Cancer. 2012;107(4):695-699.

27. Wang L, Jia J, Lin L, et al. Predictive value of hematological markers of systemic inflammation for managing cervical cancer. Oncotarget 2017;8(27):44824-44832

28. Zhang Y, Wang L, Liu Y, et al. Preoperative neutrophil-lymphocyte ratio before platelet-lymphocyte ratio predicts clinical outcome in patients with cervical cancer treated with initial radical surgery. Int $J$ Gynecol Cancer. 2014;24(7):1319-1325.

29. Mizunuma M, Yokoyama Y, Futagami M, Aoki M, Takai Y, Mizunuma $\mathrm{H}$. The pretreatment neutrophil-to-lymphocyte ratio predicts therapeutic response to radiation therapy and concurrent chemoradiation therapy in uterine cervical cancer. Int J Clin Oncol. 2015;20(5):989-996. 
30. Huang QT, Man QQ, Hu J, et al. Prognostic significance of neutrophilto-lymphocyte ratio in cervical cancer: A systematic review and metaanalysis of observational studies. Oncotarget. 2017;8(10):16755-16764.

31. Templeton AJ, McNamara MG, Šeruga B, et al. Prognostic role of neutrophil-to-lymphocyte ratio in solid tumors: a systematic review and meta-analysis. J Natl Cancer Inst. 2014;106(6):dju124.

32. Cho Y, Kim KH, Yoon HI, Kim GE, Kim YB. Tumor-related leukocytosis is associated with poor radiation response and clinical outcome in uterine cervical cancer patients. Ann Oncol. 2016;27(11):2067-2074.

33. Wang D, Wu M, Feng FZ, et al. Pretreatment neutrophil-to-lymphocyte and platelet-to-lymphocyte ratios do not predict survival in patients with cervical cancer treated with neoadjuvant chemotherapy and radical hysterectomy. Chin Med J. 2013;126(8):1464-1468.

34. Kose M, Celik F, Kose SK, Arioz DT, Yilmazer M. Could the plateletto-lymphocyte ratio be a novel marker for predicting invasiveness of cervical pathologies? Asian Pac J Cancer Prev. 2015;16(3):923-926.

35. Smith RA, Bosonnet L, Ghaneh P, et al. The platelet-lymphocyte ratio improves the predictive value of serum CA19-9 levels in determining patient selection for staging laparoscopy in suspected periampullary cancer. Surgery. 2008;143(5):658-666.

36. Acmaz G, Aksoy H, Unal D, et al. Are neutrophil/lymphocyte and platelet/lymphocyte ratios associated with endometrial precancerous and cancerous lesions in patients with abnormal uterine bleeding? Asian Pac J Cancer Prev. 2014;15(4):1689-1692.

37. Kozasa K, Mabuchi S, Komura N, et al. Comparison of clinical utilities of the platelet count and platelet-lymphocyte ratio for predicting survival in patients with cervical cancer: a single institutional study and literature review. Oncotarget. 2017;8(33):55394-55404.

38. Chen L, Zhang F, Sheng XG, Zhang SQ, Chen YT, Liu BW. Peripheral platelet/lymphocyte ratio predicts lymph node metastasis and acts as a superior prognostic factor for cervical cancer when combined with neutrophil: Lymphocyte. Medicine. 2016;95(32):e4381.

39. Nakamura K, Nishida T, Haruma T, et al. Pretreatment plateletlymphocyte ratio is an independent predictor of cervical cancer recurrence following concurrent chemoradiation therapy. Mol Clin Oncol. 2015;3(5):1001-1006.
40. Sprague BL, Trentham-Dietz A, Klein BE, et al. Physical activity, white blood cell count, and lung cancer risk in a prospective cohort study. Cancer Epidemiol Biomarkers Prev. 2008;17(10):2714-2722.

41. Tavares-Murta BM, Mendonça MA, Duarte NL, et al. Systemic leukocyte alterations are associated with invasive uterine cervical cancer. Int J Gynecol Cancer. 2010;20(7):1154-1159.

42. Haraga J, Nakamura K, Omichi C, et al. Pretreatment prognostic nutritional index is a significant predictor of prognosis in patients with cervical cancer treated with concurrent chemoradiotherapy. Mol Clin Oncol. 2016;5(5):567-574.

43. Gelband H, Sankaranarayanan R, Gauvreau CL, et al. Costs, affordability, and feasibility of an essential package of cancer control interventions in low-income and middle-income countries: key messages from Disease Control Priorities, 3rd edition. Lancet. 2016;387(10033): 2133-2144.

44. Onal C, Guler OC, Yildirim BA. Prognostic Use of pretreatment hematologic parameters in patients receiving definitive chemoradiotherapy for cervical cancer. Int J Gynecol Cancer. 2016;26(6): 1169-1175.

45. Wang YY, Bai ZL, He JL, et al. Prognostic value of neutrophil-related factors in locally advanced cervical squamous cell carcinoma patients treated with cisplatin-based concurrent chemoradiotherapy. Dis Markers. 2016;2016:1-9.

46. Zheng RR, Huang M, Jin C, et al. Cervical cancer systemic inflammation score: a novel predictor of prognosis. Oncotarget. 2016;7(12): $15230-15242$

47. Mantovani A, Allavena P, Sica A, Balkwill F. Cancer-related inflammation. Nature. 2008;454(7203):436-444

48. Balkwill F, Mantovani A. Inflammation and cancer: back to Virchow? Lancet. 2001;357(9255):539-545.

49. Paramanathan A, Saxena A, Morris DL. A systematic review and metaanalysis on the impact of pre-operative neutrophil lymphocyte ratio on long term outcomes after curative intent resection of solid tumours. Surg Oncol. 2014;23(1):31-39.
Cancer Management and Research

\section{Publish your work in this journal}

Cancer Management and Research is an international, peer-reviewed open access journal focusing on cancer research and the optimal use of preventative and integrated treatment interventions to achieve improved outcomes, enhanced survival and quality of life for the cancer patient. The manuscript management system is completely online and includes

\section{Dovepress}

a very quick and fair peer-review system, which is all easy to use. Visit http://www.dovepress.com/testimonials.php to read real quotes from published authors. 